Published in final edited form as:

Pediatrics. 2012 December ; 130(6): e1425-e1432. doi:10.1542/peds.2012-1057.

\title{
Pediatric and Adolescent Tuberculosis in the United States, 2008-2010
}

\author{
Carla A. Winston, PhD, MA and Heather J. Menzies, MD, MPH \\ Division of Tuberculosis Elimination, Centers for Disease Control and Prevention, Atlanta, \\ Georgia
}

\section{Abstract}

OBJECTIVE-We examined heterogeneity among children and adolescents diagnosed with tuberculosis (TB) in the United States, and we investigated potential international TB exposure risk.

METHODS-We analyzed demographic and clinical characteristics by origin of birth for persons $<18$ years with verified case of incident TB disease reported to National TB Surveillance System from 2008 to 2010 . We describe newly available data on parent or guardian countries of origin and history of having lived internationally for pediatric patients with TB $(<15$ years of age).

RESULTS-Of 2660 children and adolescents diagnosed with TB during 2008-2010, 822 (31\%) were foreign-born; Mexico was the most frequently reported country of foreign birth. Over half (52\%) of foreign-born patients diagnosed with TB were adolescents aged 13 to 17 years who had lived in the United States on average $>3$ years before TB diagnosis. Foreign-born pediatric patients with foreign-born parents were older (mean, 7.8 years) than foreign-born patients with US-born parents (4.2 years) or US-born patients (3.6 years). Among US-born pediatric patients, $66 \%$ had at least 1 foreign-born parent, which is $>3$ times the proportion in the general population. Only $25 \%$ of pediatric patients with TB diagnosed in the United States had no known international connection through family or residence history.

CONCLUSIONS-Three-quarters of pediatric patients with TB in the United States have potential TB exposures through foreign-born parents or residence outside the United States. Missed opportunities to prevent TB disease may occur if clinicians fail to assess all potential TB exposures during routine clinic visits.

\footnotetext{
Address correspondence to Carla A. Winston, PhD, MA, Centers for Disease Control and Prevention, 1600 Clifton Rd NE, Mailstop E-91, Atlanta, GA 30333. cwinston@cdc.gov.

The findings and conclusions are those of the authors and do not necessarily represent the views of the Centers for Disease Control and Prevention.

Both authors made substantial intellectual contributions to the study. Dr Winston acquired and had full access to all the data in the study and takes responsibility for the integrity of the data and accuracy of the data analysis. Both Drs Winston and Menzies participated in study concept and design and in interpretation of data. Dr Winston drafted the article, which Dr Menzies critically revised for important intellectual content. Both authors approved the final version.
}

FINANCIAL DISCLOSURE: The authors have indicated they have no financial relationships relevant to this article to disclose. COMPANION PAPERS: Companions to this paper can be found on pages e1433 and e1672, and online at www.pediatrics.org/cgi/doi/10.1542/peds.2011-3742 and www.pediatrics.org/cgi/doi/10.1542/peds.2012-2832. 


\section{Keywords}

emigration and immigration; public health practice; epidemiologic factors; tuberculosis; vulnerable populations

Tuberculosis (TB) among young children represents recent transmission and is a marker for TB control. ${ }^{1}$ Children and adolescents with latent TB infection also represent a pool of future disease. In the United States, routine tuberculin skin testing for TB infection is not recommended because of the low incidence of TB disease. ${ }^{1,2}$ Instead, TB risk assessment during well-child visits and other clinical encounters is encouraged. Such assessments help determine which children and adolescents may benefit from targeted testing for and treatment of latent infection to prevent development of TB disease. Validated TB risk questionnaires assess whether family members or close contacts have TB infection or disease, and whether the child was born in a TB-endemic country or has traveled outside the United States and had contact with populations where TB is endemic. ${ }^{1,3,4}$ Although health care providers may be able to assess risk factors for individual patients, these data for pediatric TB cases have been unavailable at the national level until now.

TB is a nationally notifiable disease in the United States. Cases verified according to a standard case definition ${ }^{5}$ are reported electronically by health departments to the Centers for Disease Control and Prevention (CDC). The CDC maintains the US National TB Surveillance System, which has collected detailed demographic and clinical data on TB cases since $1993 .{ }^{2}$ Beginning in 2009, data are now reported regarding whether patients have an epidemiologic link to another verified TB case, and whether cases are identified through contact investigation, targeted testing for TB infection, incidental findings in an asymptomatic patient, or evaluation of clinical signs and symptoms. Since 2009, data for pediatric patients ( $<15$ years old) also include parent or guardian countries of birth and history of having lived outside the United States. We examined characteristics of children and adolescents diagnosed with TB in the United States during 2008-2010, and results of analyses of newly available national data for pediatric and adolescent TB surveillance during 2009-2010.

\section{METHODS}

Analyses included all persons $<18$ years old with a verified case of incident TB disease reported to the CDC from January 1, 2008, through December 31, 2010, by the 50 United States and the District of Columbia. We examined TB case frequency by US Census Bureau categorization of US- or foreign-born origin (anyone not a US citizen at birth is considered foreign-born $)^{6}$ according to patient age, gender, race and ethnicity per self-report or parental report (Hispanic, or non-Hispanic white, black, Asian/Pacific Islander, American Indian/ Native Alaskan), disease site (pulmonary, extrapulmonary, or both), hierarchical TB verification criteria (ie, [1] positive culture or [2] nucleic acid amplification test for Mycobacterium tuberculosis, [3] positive smear or examination for acid-fast bacilli, [4] clinical confirmation by tuberculin skin test or interferon $\gamma$ release assay results and chest radiograph or scan, or [5] provider diagnosis in the absence of clinical results), HIV infection status (positive, negative, or not reported to TB surveillance), and TB drug 
resistance pattern (no known resistance, isoniazid resistance, or isoniazid and rifampin resistance [multidrug-resistant (MDR) TB]). We reported $P$ values comparing patient characteristics by using $\chi^{2}$ tests, or when cell sizes were $<5$, Fisher's exact tests. We compared mean age by using $t$ tests. For foreign-born children and adolescents, we examined age at US arrival and age at TB diagnosis by country of origin. Analyses were approved by the $\mathrm{CDC}$ as routine public health surveillance, which does not require institutional board review.

We analyzed data newly available in 2009-2010 regarding the primary reason persons $<18$ years were initially evaluated for TB disease and whether they had epidemiologic links to another patient with TB (Fig 1). We identified what proportion of cases among children and adolescents had identical TB strains as an epidemiologically linked case, defined as matching $M$ tuberculosis genotypes based on spoligotype and 12-locus mycobacterial interspersed repetitive units; both methods are used routinely in national TB surveillance and have $>95 \%$ discriminatory power. ${ }^{7-10}$ Matching TB genotypes may indicate that 2 cases are in the same chain of disease transmission; however, no information was available regarding whether the reported epidemiologic link was considered a source case, simultaneously exposed person, or unrelated in the chain of transmission.

For pediatric patients, we analyzed variables newly available in 2009-2010: countries of birth for parents or guardians, whether patients lived outside the United States for $>2$ months, and countries outside the United States where pediatric patients resided. These data are limited to children $<15$ years old because data on parents/guardians and international residence are only collected on this subset of patients with TB (Fig 1). We refer to parent or primary guardian as parent.

\section{RESULTS}

\section{Characteristics of Children and Adolescents Diagnosed With TB}

The number of TB cases among children and adolescents $<18$ years old in the United States declined from 977 in 2008 to 865 in 2009 and 818 in 2010. Children and adolescents comprised $7 \%$ of all TB cases reported annually.

Of children and adolescents diagnosed with TB in the United States during 2008-2010 (Fig 1), 822 (31\%) were foreign-born and 1826 (69\%) were US-born. Foreign-born patients were older (11.0 years versus 5.5 years, $P<.0001$ ) and more likely to have pulmonary disease than US-born patients ( $72 \%$ vs $67 \%$ ), who were more likely to have extrapulmonary or combined pulmonary and extrapulmonary presentation (Table 1). More than half of US-born patients (52\%) were Hispanic compared with $30 \%$ of foreign-born patients. Just over onethird (36\%) of foreign-born patients were Asian or Pacific Islander compared with 13\% of US-born patients, whereas non-Hispanic black patients made up 25\% of US-born and 30\% of foreign-born patients. A larger proportion of foreign-born patients had results reported for HIV status (59\%) than did US-born patients (39\%). Among those with known status, $1 \%$ of both US- and foreign-born children were HIV-positive $(P=.58)$. Corresponding with their older age and clinical presentation (ie, more likely to have pulmonary TB), foreign-born patients were more likely to have a positive culture than US-born patients (43\% vs $32 \%$ ). 
Over $96 \%$ of culture-positive patients had initial drug susceptibility testing results, with higher proportions of isoniazid-resistant (11\% vs $6 \%$ ) and MDR (4\% vs $1 \%)$ TB among foreign-born compared with US-born patients.

\section{Country of Origin and Age at TB Diagnosis Among Foreign-born Children and Adolescents}

Over half (52\%) of all foreign-born patients were diagnosed between age 13 and 17 years (Table 1); on average, these adolescents had lived in the United States for 3.5 years before diagnosis. Top countries of origin among foreign-born children and adolescents were Mexico (19\%), the Philippines (9\%), Ethiopia (6\%), Haiti (6\%), Burma (5\%), Somalia (5\%), Vietnam (4\%), China (4\%), and India (4\%). Children born in Mexico were generally older at TB diagnosis though younger at first arrival to the United States compared with other foreign-born patients (Fig 2). The longest average time from US arrival to TB diagnosis was 4.5 years among patients born in Mexico, followed by Somalia (3.0 years), Vietnam (2.9 years), and India (2.7 years). The shortest average times from US arrival to diagnosis were among patients from China (1.2 years), and Burma and Ethiopia, averaging < 1 year ( 0.6 years) for the latter 2 countries. Children born in Ethiopia made up a disproportionate share of foreign-born patients with TB $<1$ year of age ( 6 of 17 infants).

\section{Epidemiologic Links to Known TB Cases and Primary Reason Evaluated for TB}

Of 1680 patients with TB <18 years old in 2009-2010 (Fig 1), 201 (12\%) were epidemiologically linked to at least 1 other patient with TB in the US National TB Surveillance database. Among 188 US-born children and adolescents, 103 (55\%) were linked to a foreign-born case. Among 13 foreign-born children and adolescents, 2 (15\%) were linked to a US-born case. Patients with TB to whom children and adolescents were epidemiologically linked ranged from $<1$ to 87 years old and were predominantly Hispanic among both US-born (40\%) and foreign-born (69\%) patients. Among US-born children and adolescents, $39 \%$ of epidemiologically linked patients with TB were non-Hispanic black.

Similar to the proportion among all patients $<18$ years old, one-third (34\%) of child and adolescent patients with TB with an epidemiologic link had a positive culture for $M$ tuberculosis; among epidemiologically linked patients with TB (mostly adults), 90\% were culture positive. A total of 60 pairs of child and adolescent patients and linked case patients had genotyping results, of whom $55(92 \%)$ had identical or nearly identical TB genotypes (53 matched exactly; 2 differed by 1 position in spoligotype or 12-locus mycobacterial interspersed repetitive units).

Of US-born child and adolescent cases with epidemiologic links, 70\% were initially evaluated for TB because they were contacts of a known case, whereas $16 \%$ sought attention for symptoms (Table 2). By comparison, among US-born child and adolescent patients with TB overall, one-third (32\%) were evaluated during a contact investigation, one-third (29\%) were symptomatic, and $15 \%$ were diagnosed based on radiographic findings when TB was not initially suspected. Among foreign-born children and adolescents, similar proportions to the US-born overall were symptomatic (33\%) or identified due to incidental radiographic findings (22\%). All 13 foreign-born patients with epidemiologic links were assessed during a TB case contact investigation, compared with $8 \%$ of overall foreign-born cases; 
immigration medical examinations also yielded $8 \%$. Targeted testing programs resulted in $4 \%$ of all child and adolescent TB cases, and $19 \%$ were missing data on reason evaluated for TB.

\section{Country of Origin of Parents of Pediatric Patients With TB Aged $<15$ Years}

There were 1282 pediatric patients with TB in 2009-2010 (Fig 1), including 291 foreignborn and 991 US-born patients. Data for parent countries of birth were available for 805 patients. Among foreign-born pediatric patients, leading parent countries of birth were the United States (15\%), Mexico (12\%), the Philippines (10\%), Burma (8\%), Haiti (7\%), and Somalia (6\%). Among US-born pediatric patients, leading parent countries of birth were the United States (35\%), Mexico (29\%), Guatemala (5\%), India (4\%), Vietnam (3\%), El Salvador (3\%), and Honduras (3\%).

Among patients with data, 85\% (169 of 198) of foreign-born and 66\% (400 of 607) of USborn children with TB had at least 1 foreign-born parent (Table 3). Forty percent (40\%) of foreign-born pediatric patients with foreign-born parents were Asian, whereas $68 \%$ of USborn patients with foreign-born parents were Hispanic, and $43 \%$ of US-born patients with only US-born parents were non-Hispanic black. Among foreign-born pediatric patients with US-born parents, $62 \%$ were between age 1 and 4,72\% were black, and 15 of 29 were from Ethiopia. Foreign-born pediatric patients with foreign-born parents were older (mean, 7.8 years) than foreign-born patients with US-born parents (4.2 years) or US-born patients with a foreign-born parent (3.6 years) or only US-born parents (3.7 years; $P<.0001$ for all comparisons to foreign-born patient and foreign-born parent, no other significant differences). None of these children were reported to be positive for HIV infection; however, 51\% did not have HIV status reported. Among children with positive culture and drug susceptibility results, $17 \%$ with any foreign-born parent versus $2 \%$ of patients with only US-born parents were infected with drug-resistant TB $(P=.004)$.

\section{International Residence History Among Pediatric Patients With TB}

Data for international residence history were available for 859 patients. Among 212 foreignborn pediatric patients with data, $187(88 \%)$ reported ever having lived outside the United States for $>2$ months. For most (95\%), the only country in which children had lived outside the United States was their country of birth. The few discordant responses were suggestive of refugee or displaced person migration (eg, Somalia-Kenya). Among 647 US-born patients with responses, $81(13 \%)$ were reported to have ever lived outside the United States for $>2$ months. Most frequently named countries were Mexico $(n=31)$, India $(n=8)$, and Haiti $(n=5)$. Among 765 pediatric patients with complete data, 188 (25\%) were not foreignborn, did not have a foreign-born parent or guardian, and had never resided outside the United States for $>2$ months.

\section{DISCUSSION}

Consistent with overall declines in the United States, the number of TB cases in children and adolescents declined in 2009 and 2010 . The decline in 2009 was unusually steep and likely related to economic and immigration trends, as described elsewhere. ${ }^{11}$ Continued attention 
to TB prevention among children and adolescents is critical to sustaining these declines and accelerating progress toward achieving a TB-free generation. Our findings highlight 4 diverse groups that bear an increased burden of TB disease and in whom preventive efforts could be enhanced: (1) US-born children with foreign-born parents, (2) foreign-born children with US-born parents, (3) foreign-born adolescents, and (4) US-born children traditionally at risk for TB.

Two-thirds (66\%) of US-born pediatric patients with TB in our data have a foreign-born parent compared with $18 \%$ of the general US-born population younger than 18 years. ${ }^{12}$ Our estimate is higher than reported in a previous study, ${ }^{13}$ perhaps reflecting the increasing impact of the global burden of TB on the United States over time. The majority of foreignborn parents of US-born children with TB are from Mexico or Central America, where TB infection prevalence is higher than the United States. ${ }^{14}$ Just over half (52\%) of US-born child and adolescent patients with TB are Hispanic, compared with $19 \%$ of all US-born patients with TB; both proportions have increased in recent years. ${ }^{2,15}$ Non-Hispanic Asian and black persons are also disproportionately represented among foreign-born patients with TB and parents of patients with TB, highlighting the diversity of TB risk based on TB prevalence in countries of origin and emigration trends. ${ }^{16}$

In our data, $4 \%$ of pediatric patients with TB are foreign-born (not US citizens at birth) with only US-born parents, which may reflect international adoption. Because surveillance data do not ask about adoption, we are not able to confirm this directly. Foreign-born children should be medically evaluated before leaving their home country and upon arrival to the United States. ${ }^{17-20}$ Children who are negative for TB infection should be reexamined within 6 months of US arrival and after any subsequent exposure to TB risk. ${ }^{19-21}$

In national data, the majority of foreign-born patients younger than age 18 with TB are adolescents diagnosed after many years of residence in the United States. Foreign-born adolescents experience the greatest TB disease disparity relative to US-born persons of the same age. ${ }^{15}$ Providers should not assume that latent TB infection has been assessed or adequately treated earlier in life, and should continue to assess TB exposure during patient encounters. ${ }^{1,13,22}$

US-born children diagnosed with TB are younger than foreign-born patients, reflecting high TB rates among young children. ${ }^{15}$ Similar to national surveillance for all US-born patients with $\mathrm{TB},{ }^{2}$ pediatric patients with $\mathrm{TB}$ with only US-born parents are predominantly black $(43 \%)$. Black persons in the United States are at increased risk for TB since they are more likely to be latently infected with $\mathrm{TB}^{23}$ and have 8 times the rate of $\mathrm{TB}$ disease as white persons. ${ }^{2}$ Children and adolescents at risk for TB include both those with foreign associations and those who have no international connections, but other demographic, socioeconomic, or clinical risks for $\mathrm{TB},{ }^{13,24}$ which should be assessed during routine clinical encounters.

The fact that one-third (or more ${ }^{13,25}$ ) of children and adolescents overall are symptomatic at initial evaluation suggests missed opportunities for identifying and treating TB infection to prevent TB disease. The fact that one-fifth are identified based on incidental radiographic 
findings suggests that providers may not be "thinking TB," possibly leading to delays in care. Compared with an earlier era, our data reveal that a higher proportion of children with TB have known HIV infection status, with a lower percentage reported HIV-positive than previously. ${ }^{15}$ All persons diagnosed with TB disease or latent TB infection should receive counseling and testing for HIV. ${ }^{26}$ However, reporting of HIV results to the US National TB Surveillance System is incomplete ${ }^{2}$ and does not reflect fully data that may be available at the local or state level, which impacts the analysis of these data at the national level.

Importantly, TB drug resistance remains a concern. Children with foreign-born parents are more likely to have isoniazid resistance alone or in combination with rifampin resistance. Our findings are consistent with patterns among US- and foreign-born Hispanic populations in the United States. ${ }^{27,28}$ These data point to the importance of drug susceptibility testing among patients with TB to whom children are exposed, to gauge which children may require modified TB prophylaxis or treatment regimens. ${ }^{26,29-31}$ Similar to other studies, ${ }^{32-34}$ we found that most (92\%) child and adolescent patients with TB have identical or near-identical TB strains to a reported epidemiologically linked case. Combining contact tracing and genotyping can help identify presumed source cases, interrupt ongoing transmission, and aid treatment decisions. ${ }^{25,33-35}$

This study is subject to a number of limitations. The main limitation of our analyses using national surveillance reporting is missing data. Data completeness for new pediatric variables increased in 2010 from 2009 when reporting was first introduced; HIV reporting has also improved. We anticipate that future data will become more complete. However, we do not have detailed immigration information or full travel history to TB-endemic regions. Rather, surveillance definitions reflect Census designations for US- and foreign-born, and the time frame of $>2$ months living internationally, which does not capture shorter trips that may be important in terms of TB exposure. ${ }^{1,13,36,37}$ Moreover, we can only identify country of birth of parent or guardian, not current living situation, contact with international family or other visitors, or exposures outside of the home (eg, day care, schools, institutional settings). $24,32-34,37$

\section{CONCLUSIONS}

We estimate that three-quarters of pediatric patients with TB in the United States have potential TB exposures through foreign-born parents or residence outside the United States that might remain undiscovered if providers assess only country of birth of the child or adolescent when assessing TB risk. As surveillance data for TB risk factors among pediatric patients become more complete, combining national data with local epidemiology and clinical assessments will be important for guiding TB control for the next generation. Continued attention to TB prevention and education are needed to achieve TB elimination among children in the United States.

\section{Acknowledgments}

FUNDING: Authors were salaried by the Centers for Disease Control and Prevention. No funding was received for the study. 
We acknowledge the state and local health department staff who serve patients with TB and collect data reported in these analyses. We thank Roque Miramontes, Tom Navin, and Eleanor Click for their review of preliminary findings. We acknowledge Jan Brzuskiewicz for graphical assistance.

\title{
ABBREVIATIONS
}

\author{
CDC Centers for Disease Control and Prevention \\ MDR multidrug-resistant, resistant to isoniazid and rifampin \\ TB tuberculosis
}

\section{References}

1. American Academy of Pediatrics. Tuberculosis. In: Pickering, LK.; Baker, CJ.; Kimberlin, DW.; Long, SS., editors. Red Book: 2009 Report of the Committee on Infectious Diseases. 28. Elk Grove Village, IL: American Academy of Pediatrics; 2009. p. 680-701.

2. Centers for Disease Control and Prevention. Reported Tuberculosis in the United States, 2010. Atlanta, GA: US Department of Health and Human Services; 2011.

3. Froehlich H, Ackerson LM, Morozumi PA; Pediatric Tuberculosis Study Group of Kaiser Permanente, Northern California. Targeted testing of children for tuberculosis: validation of a risk assessment questionnaire. Pediatrics. 2001; 107(4) Available at: www.pediatrics.org/cgi/content/ full/107/4/e54

4. Cruz AT, Starke JR. Pediatric tuberculosis. Pediatr Rev. 2010; 31(1):13-25. quiz 25-26.

5. Centers for Disease Control and Prevention. [Accessed February 27, 2012] Tuberculosis (Mycobacterium tuberculosis) 2009 case definition. Available at: www.cdc.gov/osels/ ph_surveillance/nndss/casedef/tuberculosis_current.htm

6. US Census Bureau. [Accessed March 7, 2012] About foreign-born population. Available at: www.census.gov/population/foreign/about/

7. Centers for Disease Control and Prevention. New CDC program for rapid genotyping of Mycobacterium tuberculosis isolates. MMWR Morb Mortal Wkly Rep. 2005 Jan.54(2):47.

8. Centers for Disease Control and Prevention. [Accessed June 26, 2012] Tuberculosis genotyping. Available at: www.cdc.gov/tb/publications/factsheets/statistics/genotyping.htm

9. Sola C, Filliol I, Legrand E, et al. Genotyping of the Mycobacterium tuberculosis complex using MIRUs: association with VNTR and spoligotyping for molecular epidemiology and evolutionary genetics. Infect Genet Evol. 2003; 3(2):125-133. [PubMed: 12809807]

10. van Deutekom H, Supply P, de Haas PE, et al. Molecular typing of Mycobacterium tuberculosis by mycobacterial interspersed repetitive unit-variable-number tandem repeat analysis, a more accurate method for identifying epidemiological links between patients with tuberculosis. J Clin Microbiol. 2005; 43(9):4473-4479. [PubMed: 16145094]

11. Winston CA, Navin TR, Becerra JE, et al. Unexpected decline in tuberculosis cases coincident with economic recession - United States, 2009. BMC Public Health. 2011; 11:846. [PubMed: 22059421]

12. US Department of Health and Human Services, Health Resources and Services Administration, Maternal and Child Health Bureau. . Child Health USA 2008-2009. Rockville, MD: US Department of Health and Human Services; 2009. Available at: www.mchb.hrsa.gov/chusa08/ more/introduction.html [Accessed January 10, 2012]

13. Lobato MN, Sun SJ, Moonan PK, et al. Zero Tolerance for Pediatric TB Study Group. Underuse of effective measures to prevent and manage pediatric tuberculosis in the United States. Arch Pediatr Adolesc Med. 2008; 162(5):426-431. [PubMed: 18458188]

14. World Health Organization. [Accessed March 19, 2012] WHO report 2009: global tuberculosis control - epidemiology, strategy, financing (WHO/HTM/TB/2009.411). Available at: www.who.int/tb/publications/global_report/2009/en/index.html 
15. Menzies HJ, Winston CA, Holtz TH, Cain KP, Mac Kenzie WR. Epidemiology of tuberculosis among US- and foreign-born children and adolescents in the United States, 1994-2007. Am J Public Health. 2010; 100(9):1724-1729. [PubMed: 20634457]

16. Cain KP, Benoit SR, Winston CA, Mac Kenzie WR. Tuberculosis among foreign-born persons in the United States. JAMA. 2008; 300(4):405-412. [PubMed: 18647983]

17. American Academy of Pediatrics. Medical evaluation of internationally adopted children for infectious diseases. In: Pickering, LK.; Baker, CJ.; Kimberlin, DW.; Long, SS., editors. Red Book: 2009 Report of the Committee on Infectious Diseases. 28. Elk Grove Village, IL: American Academy of Pediatrics; 2009. p. 177-179.p. 181

18. Jones VF. Committee on Early Childhood, Adoption, and Dependent Care. Comprehensive health evaluation of the newly adopted child. Pediatrics. 2012; 129(1) Available at: www.pediatrics.org/cgi/content/full/129/1/e214.

19. Centers for Disease Control and Prevention. [Accessed March 10, 2012] International adoption: health guidance and the immigration process. Available at: www.cdc.gov/immigrantrefugeehealth/ adoption/class-a-conditions.html

20. Centers for Disease Control and Prevention. [Accessed March 10, 2012] Technical instructions for panel physicians. Available at: www.cdc.gov/immigrantrefugeehealth/exams/ti/panel/technicalinstructions-panel-physicians.html

21. Trehan I, Meinzen-Derr JK, Jamison L, Staat MA. Tuberculosis screening in internationally adopted children: the need for initial and repeat testing. Pediatrics. 2008; 122(1) Available at: www.pediatrics.org/cgi/content/full/122/1/e7.

22. Guh A, Sosa L, Hadler JL, Lobato MN. Missed opportunities to prevent tuberculosis in foreignborn persons, Connecticut, 2005-2008. Int J Tuberc Lung Dis. 2011; 15(8):1044-1049. [PubMed: 21740666]

23. Bennett DE, Courval JM, Onorato I, et al. Prevalence of tuberculosis infection in the United States population: the national health and nutrition examination survey, 1999-2000. Am J Respir Crit Care Med. 2008; 177(3):348-355. [PubMed: 17989346]

24. Buff AM, Sosa LE, Hoopes AJ, et al. Two tuberculosis genotyping clusters, one preventable outbreak. Public Health Rep. 2009; 124(4):490-494. [PubMed: 19618785]

25. Yeo IK, Tannenbaum T, Scott AN, et al. Contact investigation and genotyping to identify tuberculosis transmission to children. Pediatr Infect Dis J. 2006; 25(11):1037-1043. [PubMed: 17072127]

26. Blumberg HM, Burman WJ, Chaisson RE, et al. American Thoracic Society, Centers for Disease Control and Prevention and the Infectious Diseases Society. Treatment of tuberculosis. Am J Respir Crit Care Med. 2003; 167(4):603-662. [PubMed: 12588714]

27. Wells CD, Ocaña M, Moser K, Bergmire-Sweat D, Mohle-Boetani JC, Binkin NJ. A study of tuberculosis among foreign-born Hispanic persons in the US States bordering Mexico. Am J Respir Crit Care Med. 1999; 159(3):834-837. [PubMed: 10051259]

28. Centers for Disease Control and Prevention (CDC). . Characteristics of foreign-born Hispanic patients with tuberculosis - eight US counties bordering Mexico, 1995. MMWR Morb Mortal Wkly Rep. 1996; 45(47):1032-1036. [PubMed: 8965804]

29. Sneag DB, Schaaf HS, Cotton MF, Zar HJ. Failure of chemoprophylaxis with standard antituberculosis agents in child contacts of multidrug-resistant tuberculosis cases. Pediatr Infect Dis J. 2007; 26(12):1142-1146. [PubMed: 18043453]

30. Lobato MN, Jereb JA, Castro KG. Do we have evidence for policy changes in the treatment of children with latent tuberculosis infection? Pediatrics. 2009; 123(3):902-903. [PubMed: 19255018]

31. Finnell SM, Christenson JC, Downs SM. Latent tuberculosis infection in children: a call for revised treatment guidelines. Pediatrics. 2009; 123(3):816-822. [PubMed: 19255008]

32. Sun SJ, Bennett DE, Flood J, Loeffler AM, Kammerer S, Ellis BA. Identifying the sources of tuberculosis in young children: a multi-state investigation. Emerg Infect Dis. 2002; 8 (11):12161223. [PubMed: 12453345] 
33. Wootton SH, Gonzalez BE, Pawlak R, et al. Epidemiology of pediatric tuberculosis using traditional and molecular techniques: Houston, Texas. Pediatrics. 2005; 116(5):1141-1147. [PubMed: 16264001]

34. Bennett DE, Onorato IM, Ellis BA, et al. DNA fingerprinting of Mycobacterium tuberculosis isolates from epidemiologically linked case pairs. Emerg Infect Dis. 2002; 8(11):1224-1229. [PubMed: 12453346]

35. Centers for Disease Control and Prevention. Guidelines for the investigation of contracts or persons with infectious tuberculosis: recommendations from the National Tuberculosis Controllers Association and CDC. MMWR Morb Mortal Wkly Rep. 2005; 54(RR-15):1-37. [PubMed: 15647722]

36. Slopen ME, Laraque F, Piatek AS, Ahuja SD. Missed opportunities for tuberculosis prevention in New York City, 2003. J Public Health Manag Pract. 2011; 17(5):421-426. [PubMed: 21788779]

37. Lobato MN, Hopewell PC. Mycobacterium tuberculosis infection after travel to or contact with visitors from countries with a high prevalence of tuberculosis. Am J Respir Crit Care Med. 1998; 158(6):1871-1875. [PubMed: 9847280] 


\section{WHAT'S KNOWN ON THIS SUBJECT}

Foreign-born children and adolescents in the United States experience higher tuberculosis (TB) morbidity rates than US-born children and adolescents. Pediatric risk assessment should account for country of birth, contact with a known TB case, or travel to TBendemic countries.

\section{WHAT THIS STUDY ADDS}

Our study reports national data on parental/guardian countries of origin and international residence of pediatric patients with TB. Two-thirds of US-born children with TB have international family connections, and many have lived in countries with increased risk for TB acquisition. 


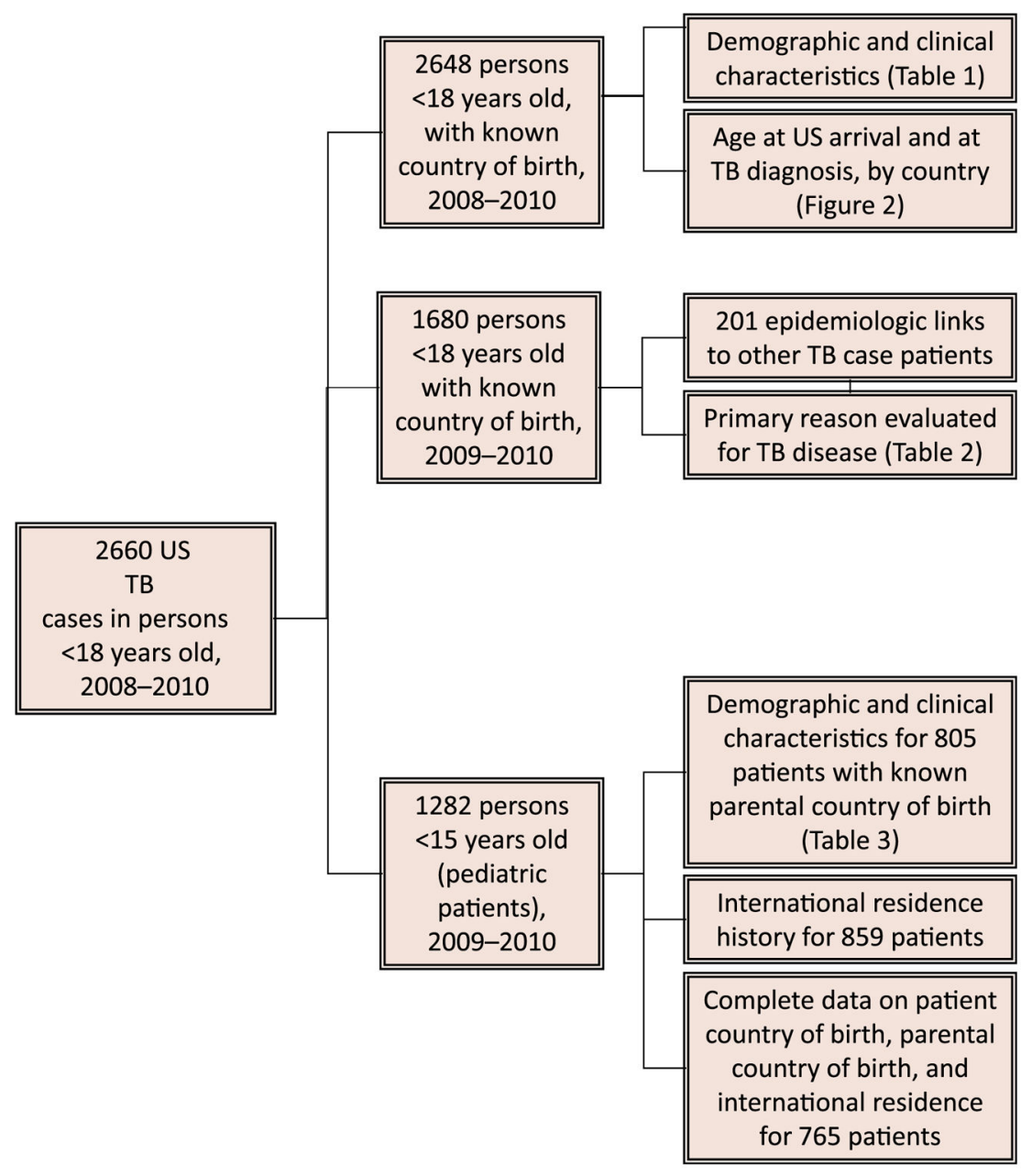

FIGURE 1.

Pediatric and adolescent TB data diagram. 


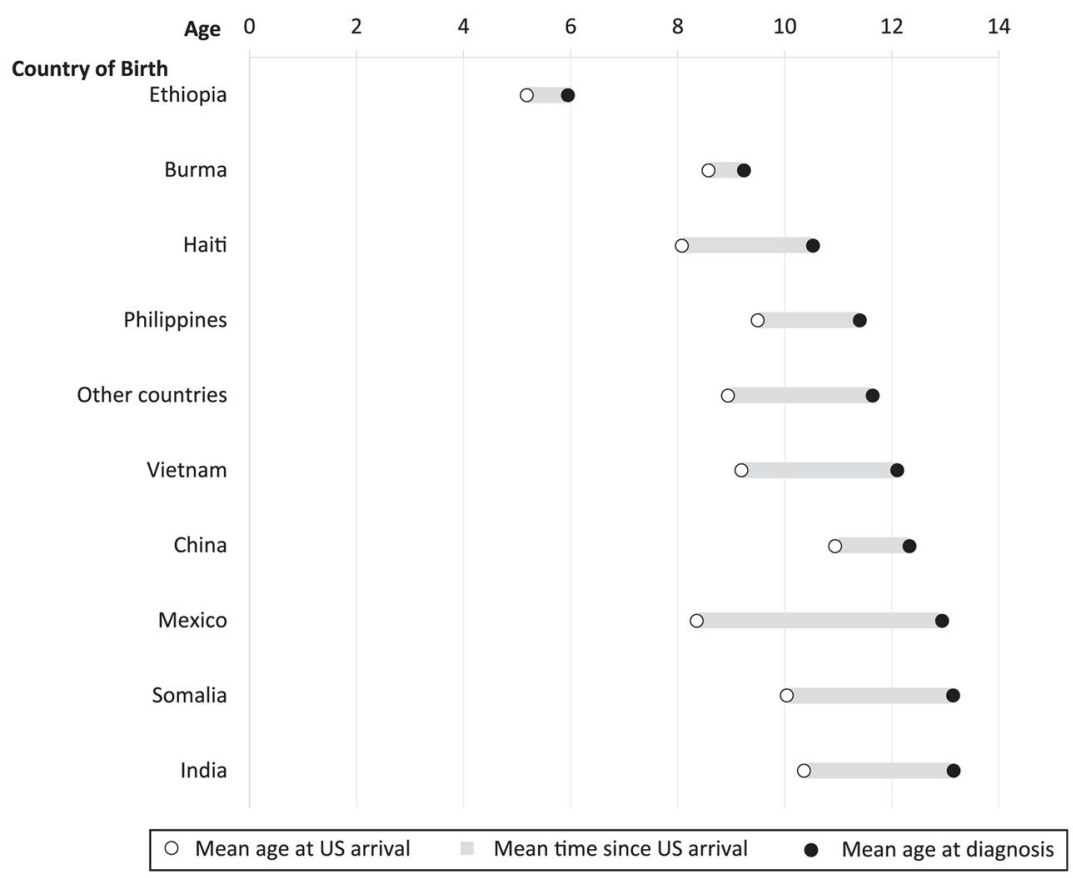

FIGURE 2.

Mean age at US arrival and at TB diagnosis among foreign-born children and adolescents, United States, 2008-2010. 
TABLE 1

Characteristics of Children and Adolescents With TB, United States, 2008-2010

\begin{tabular}{|c|c|c|c|}
\hline & US-born, $1826(100), N(\%)$ & Foreign-born, $822(100), N(\%)$ & $P$ \\
\hline Boy & $945(52)$ & $447(54)$ & .21 \\
\hline Age, y & & & $<.0001$ \\
\hline$<1$ & $268(15)$ & $17(2)$ & \\
\hline $1-4$ & $839(46)$ & $135(16)$ & \\
\hline $5-12$ & $369(20)$ & 240 (29) & \\
\hline $13-17$ & $350(19)$ & $430(52)$ & \\
\hline Race/ethnicity ${ }^{a}$ & & & $<.0001$ \\
\hline Hispanic & $947(52)$ & $242(30)$ & \\
\hline White & $139(8)$ & $38(5)$ & \\
\hline Black & $454(25)$ & $244(30)$ & \\
\hline Asian or Pacific Islander & $240(13)$ & $291(36)$ & \\
\hline American Indian or Native Alaskan & $32(2)$ & $1(0.1)$ & \\
\hline Site of disease & & & .002 \\
\hline Pulmonary & $1225(67)$ & $593(72)$ & \\
\hline Extrapulmonary & $439(24)$ & $184(22)$ & \\
\hline Both & $161(9)$ & $42(5)$ & \\
\hline TB case verification & & & $<.0001$ \\
\hline Positive culture & $592(32)$ & $353(43)$ & \\
\hline Nucleic acid amplification test & $18(1)$ & $9(1)$ & \\
\hline Positive smear absent culture & $14(0.8)$ & $2(0.2)$ & \\
\hline Clinical case & $804(44)$ & $332(40)$ & \\
\hline Provider diagnosis & $398(22)$ & $126(15)$ & \\
\hline HIV status & & & $<.0001$ \\
\hline HIV positive & $7(0.4)$ & $6(0.7)$ & \\
\hline HIV negative & 707 (39) & $476(58)$ & \\
\hline Not reported & $1112(61)$ & $340(41)$ & \\
\hline \multicolumn{4}{|l|}{ Drug resistance $^{b}$} \\
\hline None & $466(82)$ & $279(80)$ & .65 \\
\hline Isoniazid & $35(6)$ & $37(11)$ & .01 \\
\hline Isoniazid and rifampin (MDR TB $)^{c}$ & $6(1)$ & $14(4)$ & .003 \\
\hline
\end{tabular}

$N(\%)$ may vary slightly from total $(100 \%)$ due to missing data or rounding.

${ }^{a}$ Hispanic may be of any race. Excludes 20 patients of other, multiple, or unknown race responses.

${ }^{b}$ Among patients with positive culture for M tuberculosis and drug susceptibility results (571 US-born; 347 foreign-born).

${ }^{c}$ MDR TB is defined as M tuberculosis isolate with resistance to at least isoniazid and rifampin. 
TABLE 2

Primary Reason Evaluated Among Children and Adolescents With TB, United States, 2009-2010

\begin{tabular}{lcccc}
\hline & $\begin{array}{c}\text { US-born With Linked TB } \\
\text { Case, 188 (100), } \boldsymbol{N}(\boldsymbol{\%})\end{array}$ & $\begin{array}{c}\text { All US-born, 1162 (100), } \\
\boldsymbol{N}(\boldsymbol{\%})\end{array}$ & $\begin{array}{c}\text { Foreign-born With } \\
\text { Linked TB Case, 13 } \\
(\mathbf{1 0 0}), \boldsymbol{N}(\boldsymbol{\%})\end{array}$ & $\begin{array}{c}\text { All Foreign-born, } \\
\mathbf{5 1 8}(\mathbf{1 0 0}), \boldsymbol{N}(\boldsymbol{\%})\end{array}$ \\
\hline Contact investigation & $131(70)$ & $367(32)$ & $13(100)$ & $39(8)$ \\
TB symptoms & $31(16)$ & $334(29)$ & - & $169(33)$ \\
Abnormal radiograph & $17(9)$ & $170(15)$ & - & $112(22)$ \\
Targeted testing & $5(3)$ & $40(3)$ & - & $32(6)$ \\
Immigration examination & Not applicable & Not applicable & - & $43(8)$ \\
Other & $2(1)$ & $36(3)$ & - & $18(3)$ \\
Unknown & $2(1)$ & $215(19)$ & - & $105(20)$ \\
\hline
\end{tabular}

Data collection instructions were, "Select the single primary or initial reason the patient was evaluated for TB disease...the situation or reason that led to the initial suspicion that the patient might have TB disease." Definitions: contact investigation: result of a contact investigation or source case finding; TB symptoms: signs and symptoms consistent with TB (eg, prolonged persistent cough, fever, lymphadenopathy, night sweats, weight loss) if patient seeks medical attention because of symptoms not to be selected if symptoms discovered during a screening program; abnormal chest radiograph: incidental chest radiograph consistent with TB disease, independent of other choices and not the result of suspicion of TB disease; targeted testing: positive result of tuberculin skin test or interferon $\gamma$ release assay administered because the patient was specifically high risk for TB (eg, persons from area of the world with high rate of TB) or as part of a testing program focused on specific groups at risk for TB, not to be selected if another reason (eg, contact investigation, immigration medical examination, employment/administrative testing, or health care worker status) is more appropriate; immigration examination: findings of a medical examination as part of the immigration application process; other: incidental laboratory result for clinical evaluation for something other than TB (eg, bronchoscopy or autopsy) or employment or administrative TB testing such as school-based testing or testing of health care workers; unknown: reason for evaluating the patient not known. Percent totals may vary slightly from $100 \%$ due to rounding. 
TABLE 3

Characteristics of Pediatric Patients With TB in the United States, 2009-2010, by Origin of Birth and Parent Origin of Birth

\begin{tabular}{|c|c|c|c|c|}
\hline Total & $\begin{array}{l}\text { FB Patient, Any FB } \\
\text { Parent, } N=169, \%\end{array}$ & $\begin{array}{r}\text { FB Patient, Only USB } \\
\text { Parent, } N=29, \%\end{array}$ & $\begin{array}{l}\text { USB Patient, Any FB } \\
\text { Parent, } N=400, \%\end{array}$ & $\begin{array}{r}\text { USB Patient, Only USB } \\
\text { Parent, } N=207, \%\end{array}$ \\
\hline Boy & 55 & 76 & 51 & 52 \\
\hline \multicolumn{5}{|l|}{ Age, y } \\
\hline$<1$ & 2 & 3 & 17 & 18 \\
\hline $1-4$ & 25 & 62 & 55 & 52 \\
\hline $5-12$ & 54 & 34 & 24 & 25 \\
\hline $13-15$ & 18 & 0 & 5 & 5 \\
\hline \multicolumn{5}{|l|}{ Race/ethnicity } \\
\hline Hispanic & 18 & 3 & 68 & 28 \\
\hline White & 6 & 7 & 4 & 15 \\
\hline Black & 35 & 72 & 10 & 43 \\
\hline Asian or Pacific Islander & 40 & 17 & 17 & 9 \\
\hline Other/unknown & 1 & 0 & 1 & 5 \\
\hline \multicolumn{5}{|l|}{ Site of disease } \\
\hline Pulmonary & 69 & 72 & 67 & 70 \\
\hline Extrapulmonary & 25 & 21 & 23 & 23 \\
\hline Both & 5 & 7 & 10 & 7 \\
\hline \multicolumn{5}{|l|}{$\mathrm{TB}$ case verification } \\
\hline Laboratory confirmed $^{a}$ & 23 & 21 & 31 & 27 \\
\hline Clinical case & 68 & 69 & 54 & 55 \\
\hline Provider diagnosis & 9 & 10 & 16 & 18 \\
\hline \multicolumn{5}{|l|}{ HIV status } \\
\hline HIV positive & 0 & 0 & 0 & 0 \\
\hline HIV negative & 61 & 86 & 44 & 46 \\
\hline Not reported & 39 & 14 & 56 & 54 \\
\hline \multicolumn{5}{|l|}{ Drug resistance $b$} \\
\hline None & 79 & 100 & 85 & 98 \\
\hline Isoniazid & 18 & 0 & 5 & 2 \\
\hline Isoniazid and rifampin ${ }^{c}$ & 8 & 0 & 1 & 0 \\
\hline
\end{tabular}

FB, foreign-born; USB, US-born; columns may not sum to $100 \%$ due to rounding.

${ }^{a}$ Positive culture, nucleic acid amplification test, or positive smear in the absence of culture.

${ }^{b}$ Among patients with positive culture for $M$ tuberculosis and initial drug susceptibility results.

${ }^{c}$ MDR TB is defined as $M$ tuberculosis isolate with resistance to at least isoniazid and rifampin. 